\title{
GW170817: implications for the local kilonova rate and for surveys from ground-based facilities
}

\author{
M. Della Valle, ${ }^{1,2,3 \star}$ D. Guetta, ${ }^{4}$ E. Cappellaro,${ }^{5}$ L. Amati, ${ }^{6}$ M. T. Botticella, ${ }^{1}$ \\ M. Branchesi, ${ }^{7}$ E. Brocato ${ }^{\oplus},{ }^{8}$ L. Izzo ${ }^{\oplus},{ }^{2}$ M. A. Perez-Torres ${ }^{2,9}$ and G. Stratta ${ }^{10}$ \\ ${ }^{1}$ Capodimonte Astronomical Observatory, INAF-Napoli, Salita Moiariello 16, I-80131 Napoli, Italy \\ ${ }^{2}$ Instituto de Astrofísica de Andalucía (IAA-CSIC), Glorieta de la Astronomía, s/n, E-18008 Granada, Spain \\ ${ }^{3}$ International Center for Relativistic Astrophysics, Piazzale della Repubblica 2, I-65122 Pescara, Italy \\ ${ }^{4}$ Department of Physics and Optical Engineering, ORT Braude College, 21982 Karmiel, Israel \\ ${ }^{5}$ Padova Astronomical Observatory, INAF-Padova, Vicolo dell'Osservatorio 5, I-35122 Padova, Italy \\ ${ }^{6}$ INAF-IASF, Sezione di Bologna, via Gobetti 101, I-0129 Bologna, Italy \\ ${ }^{7}$ Gran Sasso Science Institute, Via Michele Jacobucci, 2, I-67100 L'Aquila AQ, Italy \\ ${ }^{8}$ Roma Astronomical Observatory, INAF-Roma, via di Frascati, 33, I-00040 Monteporzio Catone, Italy \\ ${ }^{9}$ Departamento de Fisica Teorica, Facultad de Ciencias, Universidad de Zaragoza, E-50009 Zaragoza, Spain \\ ${ }^{10}$ Universitá degli studi di Urbino Carlo Bo Dipartimento di Scienze di Base e Fondamenti, Via S. Chiara 27, I-61029 Urbino, Italy
}

Accepted 2018 September 5. Received 2018 September 4; in original form 2018 July 13

\begin{abstract}
We compute the local rate of events similar to GRB 170817A, which has been recently found to be associated with a kilonova $(\mathrm{KN})$ outburst. Our analysis finds an observed rate of such events of $R_{\mathrm{KN}} \sim 352_{-281}^{+810} \mathrm{Gpc}^{-3} \mathrm{yr}^{-1}$. After comparing at their face values this density of sGRB outbursts with the much higher density of binary neutron star (BNS) mergers of $1540_{-1220}^{+3200} \mathrm{Gpc}^{-3} \mathrm{yr}^{-1}$, estimated by LIGO-Virgo collaboration, one can conclude, admittedly with large uncertainty that either only a minor fraction of BNS mergers produces short gammaray bursts (sGRB)/KN events or the sGRBs associated with BNS mergers are beamed and observable under viewing angles as large as $\theta \lesssim 40^{\circ}$. Finally, we provide preliminary estimates of the number of sGRB/KN events detected by future surveys carried out with present/future ground-based/space facilities, such as LSST, VST, ZTF, SKA, and THESEUS.
\end{abstract}

Key words: gravitational waves - gamma-ray burst: general-supernovae: general.

\section{INTRODUCTION}

In the last decades, binary neutron star (BNS) systems have been targets of interest because of their direct link with some of the most relevant topics of modern astrophysics, such as the indirect confirmation of the existence of gravitational waves (GWs) through radio observations (Hulse \& Taylor 1975), the predicted connection with short gamma-ray bursts (sGRBs; Eichler et al. 1989) and their direct observations from $X$ and $\gamma$ satellites e.g. (Gehrels et al. 2005), and their detection as sources of GWs (Abbott et al. 2017a,b). During a BNS merger, some sub-relativistic ejecta of mass $\sim 10^{-3}-10^{-2}$ $\mathrm{M}_{\odot}$ are thrown out along the orbital plane at a modest fraction of the speed of light, $\beta=v / c=0.1-0.3$ and rapid neutron capture in the sub-relativistic ejecta (e.g. Lattimer \& Schramm 1976; Metzeger et al. 2010; Tanaka \& Hotokezaka 2013) is hypothesized to produce a $\mathrm{KN}$, an optical and near-infrared signal lasting hours to weeks (Li \& Paczynski 1998; Tanvir et al. 2013; Pian et al. 2017; Smartt et al. 2017) powered by radioactive decay. Fi- nally, this sub-relativistic ejecta transfer most of their kinetic energy $\left(\sim 10^{50}-10^{51} \mathrm{erg}\right)$ to the shocked ambient medium.

The rate of BNS mergers can be constrained in several ways: by modelling the evolution of binary systems through populations synthesis simulations (e.g. Bogomazov, Lipunov \& Tutukov 2007; Sadowski et al. 2008; Benacquista \& Downing 2013; Ziosi et al. 2014; Dominik et al. 2015; Belczynski et al. 2016; Chruslinska et al. 2018; Mapelli \& Giacobbo 2018; Kruckow et al. 2018), by the direct measurements of coalescence rates of BNS in the Milky Way from pulsar observations (Narayan et al. 1991; Kalogera et al. 2004), from the cosmic abundance of r-process elements such as Europio (Matteucci et al. 2014; Vangioni et al. 2016; Beniamini, Hotokezaka $\&$ Piran 2016), and from gravitational wave observations (Abbott et al. 2017b). However, to date, both BNS mergers and sGRBs rates are poorly constrained (see Tables 1 and 2). Moreover, it is not yet clear whether all BNS mergers produce sGRBs/KN events or only a fraction of them is able to do it (Belczynski et al. 2008). A lower limit to the measurement of the BNS merging rate can be inferred by measuring the frequency of occurrence of sGRBs, which are believed to occur during the coalescence of two neutron stars (NSs; Eichler et al. 1989; Berger 2014; D’Avanzo 2015). In this paper, 
Table 1. BNS merging rate $\left(\mathrm{Gpc}^{-3} \mathrm{yr}^{-1}\right)$.

\begin{tabular}{lccl}
\hline $\begin{array}{l}\text { Central } \\
\text { value }\end{array}$ & $\begin{array}{c}\text { Confidence } \\
\text { interval }\end{array}$ & $\begin{array}{c}\text { Confidence } \\
\text { level }\end{array}$ & \multicolumn{1}{c}{ Ref. } \\
\hline 800 & $140-2800$ & 95 per cent & Kalogera et al. (2004) \\
316 & $100-1000$ & - & Belczynski et al. (2008) \\
866 & $500-1500$ & - & Petrillo et al. (2013) \\
1540 & $320-4740$ & 90 per cent & Abbott et al. (2017b) \\
- & $300-1200$ & - & Chruslinska et al. (2018) \\
1109 & $269-3981$ & 68 per cent & Jin et al. (2018) \\
\hline
\end{tabular}

Table 2. sGRBs rate $\mathrm{Gpc}^{-3} \mathrm{yr}^{-1}$ per $f_{\mathrm{b}}^{-1}=1$.

\begin{tabular}{lcl}
\hline Central value & Confidence interval & \multicolumn{1}{c}{ Ref. } \\
\hline 10 & $5-18$ & Guetta \& Piran (2006) \\
0.6 & $0.3-9$ & Guetta \& Piran (2006) \\
0.11 & $0.07-0.18$ & Guetta \& Piran (2005) \\
40 & $10-5 \times 10^{5}$ & Nakar et al. (2006) \\
30 & $10-80$ & Guetta \& Piran (2006) \\
8 & $4-48$ & Guetta \& Piran (2006) \\
2 & $1-4$ & Guetta \& Stella (2009) \\
8 & $5-13$ & Coward et al. (2012) \\
40 & not given & Petrillo et al. (2013) \\
16.3 & $8.1-32.6$ & Jin et al. (2015) \\
11 & $4-74$ & Fong et al. (2015) \\
0.8 & $0.65-1.1$ & Ghirlanda et al. (2016) \\
0.2 & $0.13-0.24$ & Ghirlanda et al. (2016) \\
4.1 & $2.7-5.9$ & Wanderman \& Piran (2015) \\
\hline
\end{tabular}

we show that the discovery of the electromagnetic counterpart of GW 170817 (Abbott et al. 2017b, and reference therein) can help to significantly constrain this broad range of uncertainty. On the basis of this new rate, we provide preliminary estimates of the number of $\mathrm{KNe}$ detected in surveys carried out by present and next generation ground-based facilities/space missions, such as LSST, VST, ZTF, SKA, and Theseus.

\section{THE RATE OF BNS MERGING}

The rate of expected merging events has been estimated based on the observed galactic population of double NS binaries containing a radio pulsar (Narayan et al. 1991; Phinney 1991; Kalogera et al. 2001; Burgay et al. 2003). A reference number for the BNS merger rate in the Galaxy is $\sim 80_{-60}^{+200} \mathrm{Myr}^{-1}$, which can be converted to $800_{-600}^{+2000} \mathrm{Gpc}^{-3} \mathrm{yr}^{-1}$ for a galaxy number density of $10^{-2} \mathrm{Mpc}^{-3}$ (Kalogera et al. 2004). This rate has been recently revised by Chruslinska et al. (2018; see Table 1). Population synthesis studies of binary systems give results consistent with the above rates (Belczynski et al. 2002, 2007; Perna \& Belczynski 2002). Taking into account the BNS merger rate coming from the detection of GW 170817 (Abbott et al. 2017a) and the design sensitivity of Advanced LIGO and Virgo, gravitational signals from BNS mergers are expected to be detected at a rate of one every 4-90 d (Abbott et al. 2018).

\section{THE RATE OF SGRBS}

In this section, we review the estimates of the local rate of sGRBs. In spite of many efforts, the rate of sGRBs is still uncertain within a factor $\sim 10^{3}$, as it appears from an inspection of Table 2. There are many parameters that affect the estimate of the rate of sGRBs, such as (i) the shape of the luminosity function assumed for the sGRBs, (ii) the minimum of the luminosity function, (iii) the redshift distribution assumed for the sGRBs, (iv) the time-delay distribution of the merging time (from the formation of the NS to their merging), and (v) the beaming factor. ${ }^{1}$ Among these, the minimum of the sGRB Luminosity function, $L_{\min }$, is the parameter that mostly affects the local rate, as it can be clearly seen in the equation next that has been derived by Guetta \& Stella (2009):

$R_{\mathrm{sGRB}} \sim\left(\frac{R_{0}}{\mathrm{Gpc}^{-3} \mathrm{yr}^{-1}}\right)\left(\frac{L_{\mathrm{min}}}{10^{49}}\right)^{-0.5}$,

where $R_{0}$ is the central value given in Table 2. In equation (1), it is assumed that the luminosity function of sGRBs can be parametrized by a broken power law. The power-law index for the low-luminous part that best reproduced the data is $\alpha \sim 0.5$ (Guetta \& Piran 2005). Several authors have tried to estimate the local rate of sGRBs using the observed peak flux distribution and redshift distribution of the sGRBs, but still the uncertainties are large.

\subsection{Rates smaller than $1 \mathrm{Gpc}^{-3} \mathrm{yr}^{-1}$}

In this sub-section, we describe briefly the method used by different authors to derive the local rate of sGRBs. Guetta \& Piran (2005) fitted the properties of a simulated sGRB population, described by the parametric luminosity and redshift distribution, to a set of observational constraints derived from the population of sGRBs detected by BATSE. On the other hand, BATSE was insensitive to low-luminous GRBs and in view of equation (1), this means to increase the value of $L_{\min }$ and to decrease the local sGRB rate. Ghirlanda et al. (2016) find a similar result after selecting a 'bona fide' sample of 211 Fermi-GBM bursts with a peak flux larger than 5 $\mathrm{ph} \mathrm{cm}^{-2} \mathrm{~s}^{-1}$ aimed at avoiding the incompleteness effects affecting observations close to the detection threshold. By performing this selection, Ghirlanda et al. (2016) consider only the bright sGRBs $\left(\gtrsim 10^{50} \mathrm{erg}\right.$ ) that correspond to increase $L_{\min }$ in equation (1) and therefore to decrease the local sGRB rate. Both Guetta \& Piran (2005) and Ghirlanda et al. (2016) assume that sGRBs follow the star formation rate (SFR).

\subsection{Rates larger than $1 \mathrm{Gpc}^{-3} \mathrm{yr}^{-1}$}

Several authors find a larger local rate for the sGRBs performing the same analysis of Guetta \& Piran (2005) but considering different sample of sGRBs. Guetta \& Piran (2006) consider a sample of sGRBs detected by Swift. The threshold of Swift is lower than BATSE allowing the detection of low-luminous sGRBs. As it is clear from equation (1), the lowering of $L_{\min }$ implies an increase in the local rate. If sGRBs are linked to BNS mergers (see e.g. Narayan et al. 2001), the sGRB rate should be given by the convolution of the SFR with the distribution $\operatorname{Pm}(\tau)$ of the merging time $\tau$ of the binary system (Piran 1992; Ando 2004). Considering different time-delay distributions and assuming $L_{\min } \sim 10^{49} \mathrm{erg}$. Guetta \& Piran (2006) find a range of local rate of $R_{0}=8-30 \mathrm{Gpc}^{-3} \mathrm{yr}^{-1}$. The different values reported in table 2 of Guetta \& Piran (2006) correspond to different sGRB redshift and time-delay distributions considered in the paper. These values can be increased if we decrease the minimum luminosity function assumed, as it is apparent from Fig. 1. The form of the luminosity function also affects the local rate of sGRBs (Nakar et al. 2006). The latter authors use a

${ }^{1} f_{\mathrm{b}}^{-1}=1-\cos \theta$, where $\theta$ is the jet half-opening angle. 


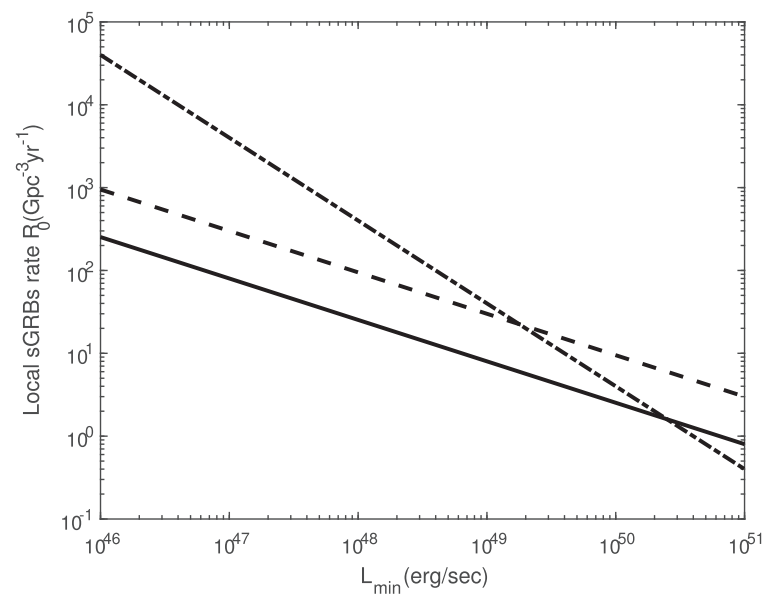

Figure 1. The 'observed' local rate of sGRBs as a function of the minimum luminosity as computed by Guetta \& Piran (2006). The rates for a uniform distribution in the delay time (solid line) and strong evolution in the delay time (dashed line) are reported. The dash-dotted line is derived considering equation (2) of Nakar et al. (2006).

power-law function for the luminosity function instead of a broken power law:

$R_{\mathrm{sGRB}} \sim\left(\frac{R_{0}}{40 \mathrm{Gpc}^{-3} \mathrm{yr}^{-1}}\right) f_{\mathrm{b}}^{-1}\left(\frac{L_{\mathrm{min}}}{10^{49}}\right)^{-1}$,

and this implies a higher local rate and stronger dependence on the lower value of the luminosity function with respect to equation (1). Other authors, such as Coward et al. (2012), avoid using an sGRB luminosity function models for progenitor rate evolution and a beaming angle distribution. Instead, they focus on observed and measured parameters that take into account selection effects that modify the Swift detection sensitivity to sGRBs. When considering the whole Swift sGRB sample, Wanderman \& Piran (2015) find that it is composed of two populations: one group that are delayed relative to the global SFR with a typical delay time of a 3-4 Gyr (depending on the SFR model) and a second group that follow the SFR with no delay. These two populations are in very good agreement with the division of sGRBs to non-Collapsars and Collapsars suggested by Bromberg et al. (2013). They find a local rate for the non-Collapsar sGRBs given by

$R_{\mathrm{SGRB}} \sim\left(\frac{R_{0}}{4.1 \pm 0.5 \mathrm{Gpc}^{-3} \mathrm{yr}^{-1}}\right) f_{\mathrm{b}}^{-1}\left(\frac{L_{\mathrm{min}}}{5 \times 10^{49}}\right)^{-0.95}$.

Guetta \& Stella (2009) consider the possibility that a fraction of sGRBs comes from the merging of dynamically formed binaries in globular clusters and infer the corresponding GW event rate that can be detected with Advanced LIGO/Virgo. In this paper, the authors show that a substantial fraction of sGRBs may occur at low redshifts, where the merging of systems formed in globular clusters through dynamical interactions is expected. They find, under this hypothesis, a merging rate larger than what expected if sGRBs come only from the merging of primordial NSs (Sadowski et al. 2008; Benacquista \& Downing 2013; Ziosi et al. 2014; Dominik et al. 2015; Belczynski et al. 2016; Chruslinska et al. 2018), of the order of $\sim 4 \mathrm{Gpc}^{-3} \mathrm{yr}^{-1}$. However, it seems that GW170817 came from an isolated binary system (Levan et al. 2017).
Table 3. Upper limits on $\mathrm{KN}$ rate.

\begin{tabular}{lccc}
\hline \multicolumn{1}{c}{ Rate } \\
Survey & $\mathrm{Gpc}^{-3} \mathrm{yr}^{-1}$ & c.l. & Ref. \\
\hline ATLAS & $<3.0 \times 10^{4}$ & 95 percent & (Smartt et al. 2017) \\
DES & $<2.4 \times 10^{4}$ & 90 per cent & (Doctor et al. 2017) \\
DLT40 & $<9.9 \times 10^{4}$ & 90 percent & (Yang et al. 2017) \\
\hline
\end{tabular}

\section{UPPER LIMITS TO THE KN RATE FROM OBSERVATIONS}

In the last decade, several sky surveys have been designed for the detection of different classes of transients such as Supernovae, Asteroids, Novae etc. Some of them as ATLAS (Tonry 2011), DES (Doctor et al. 2017), and DLT40 (Yang et al. 2017) have been used to constrain the rate of $\mathrm{KN}$ events. In Table 3, we report the estimates provided by different teams.

\section{THE RATE OF GRB 170817A-LIKE EVENTS}

GRB 170817A and GW170817 events were detected by FermiGBM (Fermi-GBM 2017) and INTEGRAL, and by Advanced LIGO and Advanced Virgo experiment. The two events, the binary coalescence and the sGRB, were detected $\sim 1.7 \mathrm{~s}$ apart (Abbott et al. 2017a; Savchenko et al. 2017). About $11 \mathrm{~h}$ later, the optical counterpart was discovered by Coulter et al. (2017) in the outskirts of the early-type galaxy NGC 4993, located at $\sim 41 \mathrm{Mpc}$ (Cantiello et al. 2018). Within $1 \mathrm{~h}$ and before the announcement of discovery, five other teams reported independent detection of the optical transients (Arcavi et al. 2017; Cowperthwaite et al. 2017; Lipunov et al. 2017; Tanvir et al. 2017; Valenti et al. 2017).

In the following, we will use the detection of an sGRB/KN event within $41 \mathrm{Mpc}$ to constrain the rate of such sources. The GRB light curve shows a weak short pulse with a duration $T_{90}$ of about $2 \mathrm{~s} \mathrm{(50-}$ $300 \mathrm{keV}$ ). The time-averaged spectrum is well fit by a power-law function with an exponential high-energy cut-off. The power-law index is $\alpha=-0.89 \pm 0.5$. The 1.024-s peak photon flux in the 50$300 \mathrm{keV}$ band is $F \sim 0.74 \mathrm{ph} \mathrm{s}^{-1} \mathrm{~cm}^{-2}$. This flux can be compared with the GBM peak flux threshold of $F_{T} \sim 0.50 \mathrm{ph} \mathrm{s}^{-1} \mathrm{~cm}^{-2}$, then deriving the maximum distance $\left(D_{\max }=49 \mathrm{Mpc}\right)$ to which the event could be detected, corresponding to the maximum volume $\left(V_{\max }=\right.$ $4.9 \times 10^{-4} \mathrm{Gpc}^{-3}$ ). After considering the Fermi-GBM (similar to the INTEGRAL/SPI-ACS) a low-orbit all-sky monitor (e.g. Meegan et al. 2009), with a sky coverage of $S_{\text {cov }} \sim 0.64$ and the number of years of operation $(T \sim 9 \mathrm{yr})$ of the GBM detector, the rate of sGRBs similar to the 170817A is

$R_{\mathrm{sGRB}}=\frac{1}{V_{\max }} \frac{1}{S_{\mathrm{cov}}} \frac{1}{T} \sim 352 \mathrm{Gpc}^{-3} \mathrm{yr}^{-1}$.

The range of uncertainty on this rate is obviously large, however, in view of the figures reported in Tables 2 and 3, it still provides interesting clues. On the basis of Poissonian statistics applied to one positive detection (Gehrels 1986), we derive an 'observed' sGRB-KN rate of $352_{-281}^{+810} \mathrm{Gpc}^{-3} \mathrm{yr}^{-1}$ that represents a substantial lower rate than the upper limits derived from the surveys reported in Table 3.

\section{DISCUSSION}

To convert the 'observed' rate into the 'true' rate of events, one has to correct the observed number for the beaming factor $f_{\mathrm{b}}^{-1}$. Unfortunately, the value of $f_{\mathrm{b}}^{-1}$ is not well known, ranging from 
values as high as $f_{\mathrm{b}}^{-1}=700-180$ corresponding to angles as small as $\theta=3^{\circ}-6^{\circ}$ (Ghirlanda et al. 2016), to intermediate values of $f_{\mathrm{b}}^{-1}=30-40$ corresponding to moderately wide angles of $\theta \sim 15^{\circ}$ (Petrillo et al. 2013; Fong et al. 2015) up to small beaming factors $f_{\mathrm{b}}^{-1} \sim 10$ for angle as broad as $\theta \sim 30^{\circ}$ (Rezzolla et al. 2011; Granot et al. 2017).

By comparing at their face values the density of BNS mergers and GRB 170817A-like events, i.e. $1540_{-1220}^{+3200} \mathrm{Gpc}^{-3} \mathrm{yr}^{-1}$ (Abbott et al. 2017a) versus 352 events $\mathrm{Gpc}^{-3} \mathrm{yr}^{-1}$, we find a range of values for the angles from which an sGRB is observable of $\sim 10^{\circ}-40^{\circ}$ in good agreement with most theoretical predictions (e.g. Lazzati et al. 2017). However, the geometry of the outflow of GRB 170817A is still a matter of discussion. The radio and X-rays that followed GW170817 are unlike any afterglow observed before, showing a gradual rise over $\sim 100 \mathrm{~d}$. They resemble the radio flare predicted long ago to follow BNS mergers. This emission arises from the interaction of the merger outflow with the external medium (Nakar \& Piran 2011). Recently, Nakar \& Piran (2018) have considered this model to explain the X-radio observations of GW170817, and they also infer that we have observed an on-axis emission of a 'structured' jet, a conclusion that might appear in mild disagreement from that derived by Troja et al. (2017), who support the idea of an offaxis view for GRB 170817A. Very recently Mooley et al. (2018) have found that the late-time emission of GRB 170817A is dominated by a narrowly collimated jet, characterized by an opening angle of $\theta \sim 5^{\circ}$, and they conclude that the emission from the jet was likely observed from a viewing angle of $\sim 20_{-5}^{+5}$.

If we assume the distance of $\sim 200 \mathrm{Mpc}$ as a plausible threshold for future detection of GWs associated with BNS mergers in the advanced LIGO/Virgo experiments (Abbott et al. 2018), we expect to detect within the corresponding volume of $3.4 \times 10^{-2} \mathrm{Gpc}^{-3}$,

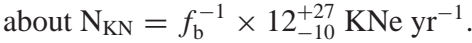

\section{KILONOVAE IN FUTURE LSST, VST, ZTF, SKA, AND THESEUS SURVEYS}

The relatively high rate of sGRB '170817A-like' that we have measured with this paper, from dozens to hundredths of events per $\mathrm{Gpc}^{3}$ per year, suggests that future optical sky surveys, designed with an appropriate observational strategy, will be able to detect a significant number of KNe without GRB or GW triggers. In the following, we will provide some preliminary estimates for the $\mathrm{KNe}$ detections from LSST, VST, ZTF, SKA, and THESEUS surveys.

\subsection{LSST}

We assume a magnitude limit of $R \sim 24$ for a typical $2 \times 15 \mathrm{~s}$ visit and $20000 \mathrm{deg}^{2}$ patrolled in $1 \mathrm{yr}$ (LSST White Paper https: //www.lsst.org/scientists/scibook) and $M_{R} \sim-16$ (Valenti et al. 2017) for the absolute magnitude at maximum of the $\mathrm{KN}$ associated with GRB 170817A. From these data, we derive a sampled volume of $\sim 2.1 \mathrm{Gpc}^{3} \mathrm{yr}^{-1}$ and therefore a number of $\mathrm{KN}$ detections of $\eta \times$ 150-2440 being $\eta$ the factor that accounts for the 'efficiency' of the survey, which depends on several parameters, such as control time (i.e. a quantity that depends on the survey cadence and the photometric time-scale evolution of the transient, see Cappellaro et al. (2015), sky conditions, technical downtime, and scheduling constraints. After an assumption of $\eta \sim 50$ per cent, we find that the optical monitoring of the sky with LSST can discover $\sim 75-1220$ $\mathrm{KN}$ events (GW170817-like) per year within $z \sim 0.25$.
Table 4. Expected blind detections of KNe from planned SKA Generic Continuum Extragalactic Surveys.

\begin{tabular}{lcccc}
\hline SKA survey & $\begin{array}{c}\text { Area } \\
\left(\mathrm{deg}^{2}\right)\end{array}$ & $\begin{array}{c}\mathrm{rms} \\
\left(\mu \mathrm{Jybam}^{-1}\right)\end{array}$ & $\begin{array}{c}\text { Sampled Volume } \\
\mathrm{Gpc}^{3} \mathrm{yr}^{-1}\end{array}$ & $\begin{array}{c}N_{\mathrm{det}} \\
\left(\mathrm{Gpc}^{-3} \mathrm{yr}^{-1}\right)\end{array}$ \\
\hline SKA1-Mid-A & 31000 & 5.0 & $17 \times 10^{-4}$ & $0.61_{-0.36}^{+1.39}$ \\
SKA1-Mid-B & 500 & 0.9 & $3.6 \times 10^{-4}$ & $0.13_{-0.08}^{+0.29}$ \\
SKA1-Mid-C & 20 & 0.24 & $1.1 \times 10^{-4}$ & $0.04_{-0.02}^{+0.08}$ \\
\hline
\end{tabular}

\subsection{VST}

After assuming a limiting magnitude of the survey of $R \sim 22.5$ in $t_{\text {exp }} \sim 1$ min (Brocato et al. 2018; Grado et al. 2018) and $M_{R} \sim$ -16 (Valenti et al. 2017) for the absolute magnitude at maximum of a 170817-like KN, and a patrolled field of $10000 \mathrm{deg}^{2}$ we find a sampled volume of $0.13 \mathrm{Gpc}^{-3}$, which implies, for $\eta=0.50$, a

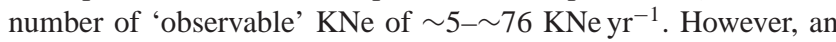
observational strategy based on a survey devoted to the continuous patrolling of known galaxy clusters within hundreds of Mpc would increase the number of KN detections. For example, with a field of view of $\sim 1 \mathrm{deg}^{2}$, VST can monitor a large sample of galaxies $(\geq 70)$ of the Hydra cluster with only four pointings. Considering a slightly different point of view, VST is expected to play a key role during the next $\mathrm{O} 3$ observing run of LIGO/Virgo collaboration (LVC). In fact, the $\mathrm{O} 3$ network of three interferometers is expected to reduce the uncertainties of the localization of GW events to few tens of square degrees with respect with previous runs. This allows VST to cover $\geq 90$ per cent of the LVC skymap probability largely increasing the efficiency in discovering $\mathrm{KN}$ candidates and in providing observational constraint on the rate of nearby $(\leq 200 \mathrm{Mpc})$ $\mathrm{KN}$ events.

\subsection{ZTF}

The Zwicky transient facility (ZTF) is a new-generation timedomain survey currently operating at the Palomar Observatory. We assume a magnitude limit of $R \sim 20.5$ for a typical exposure of $30 \mathrm{~s}$ (Bellm \& Kulkarni 2017) and $20000 \mathrm{deg}^{2}$ of sky continuously patrolled ${ }^{2}$ and $\mathrm{M}_{R} \sim-16$ for the absolute magnitude at maximum of a KN (Valenti et al. 2017). We derive a patrolled volume of $\sim 1.6 \times 10^{-2} \mathrm{Gpc}^{3}$, and therefore a number of $\mathrm{KN}$ detections of

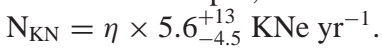

\subsection{SKA}

The square kilometre array (SKA) will survey large areas of the sky with close to $\mu \mathrm{Jy}$ sensitivities, which in principle would allow also to detect KNe from the already programmed SKA surveys. For the sake of simplicity, we discuss here three continuum generic surveys planned with the SKA1 (see Table 4). For each survey, we assume that the sampled area and the rms are obtained after $1 \mathrm{yr}$ of observations, and $3 \times 10$ min visits of the same field to reach the quoted sensitivity. We assume that the peak of brightness of GW170817 at $2.5 \mathrm{GHz}$ was $S_{v} \approx 100 \mu \mathrm{Jy}$ (e.g. Margutti et al. 2018), corresponding to a monochromatic radio luminosity of $L_{v} \approx 2.0 \times 10^{26} \mathrm{erg} \mathrm{s}^{-1} \mathrm{~Hz}^{-1}$ at the distance of its host galaxy. GW170817 peaked in the radio (at $\sim 2.5 \mathrm{GHz}$ ) around day 163 . Given the relatively standard radio synchrotron behaviour of the $\mathrm{KN}$ in GW170817, the peak at the nominal frequency of SKA of

\footnotetext{
${ }^{2}$ http://www.ztf.caltech.edu/page/technical
} 
$1.7 \mathrm{GHz}$ is expected to occur around day $\sim 163 \times(2.5 / 1.7) \approx 240 \mathrm{~d}$. Therefore, three visits over a year are enough to reliably detect any new $\mathrm{KN}$ in the radio. Hence, the factor $\eta$ that accounts for the efficiency of the survey can be safely assumed to be very close to one. However, the sensitivity of the radio surveys forces us to restrict the search for $\mathrm{KNe}$ only inside the 'local' Universe. After assuming a $5 \sigma$ threshold for a bona fide detection in a blind survey, the maximum distance to which we can confidently detect new objects is of $\approx 82,193$, and $373 \mathrm{Mpc}$ for SKA1-Mid-A, SKA1-Mid-B, and SKA1-Mid-C, respectively. The volumes sampled by the planned SKA1 surveys after $1 \mathrm{yr}$ are just a tiny fraction of a $\mathrm{Gpc}^{3}$ (see Table 4). For a KN rate of $352_{-281}^{+810} \mathrm{Gpc}^{-3} \mathrm{yr}^{-1}$ (see Section 5), the expected rate of KNs to be detected by blind radio surveys such as the ones described above is therefore quite meagre. The values reported in column 5 of Table 4 are in fact very similar since the number of detected $\mathrm{KNe}$ scales are as follows (Pérez-Torres et al. 2015): $N_{\text {det }} \propto D_{\text {mas }}^{3} / \Omega \propto \sigma_{v}^{-3 / 2} / \Omega$, where $\sigma_{v}$ is the sensitivity of the survey and $\Omega$ the angular area covered. Plugging in the numbers for each of those surveys, we see that only the SKA1-Mid-A offers some chances of getting at most one (blind) detection of a $\mathrm{KN}$ after 1 yr of observations. SKA1-Mid-B and SKA1-Mid-C, despite being significantly more sensitive than SKA1-Mid-A, cannot compensate the small area covered by those surveys.

\subsection{THESEUS}

The Transient High-Energy Sky and Early Surveyor (THESEUS) space mission (Amati et al. 2018), under study by ESA for a possible launch in 2030-2032, would be a perfect GRB and transients machine, capable of providing detection, accurate localization, redshift, and characterization of any class of GRBs and, more in general, most classes of transient sources. In particular, THESEUS will provide three observational channels for the e.m. counterparts of BNS and NS-BH mergers: the associated sGRB, the possible soft X-ray emission produced after the merging, the KN emission (Stratta et al. 2018). The on-board infrared telescope (IRT) will have a limiting magnitude of $m_{H}=20.6$ for $300 \mathrm{~s}$ of exposure. When compared with the absolute magnitude at a maximum of 170817-like KN $M_{H}=-15.5$ (Stratta et al. 2018), we find a maximum detection distance of $\sim 0.2$ Gpc. Based on these expected performances and the rate per co-moving volume and year derived above, we estimate that THESEUS/IRT can detect in $1 \mathrm{yr}$ up to $\sim 2-40$ sGRB-KN events 170817-like. The IRT will perform automatic follow-up observations of the gamma-ray and X-ray sources detected and localized by the soft X-ray imager (SXI, 1 sr FOV) and X-Gamma-Ray imaging spectrometer (XGIS, FOV > 2sr). Thus, the IRT detection rate of 170817-like KNe quoted above, should take into account the SXI and XGIS detection rate of the high-energy counterpart up to $0.2 \mathrm{Gpc}$ of the same events. The luminosities and time-scales of the predicted X-ray emission from BNS mergers are still very uncertain, and for GW170817 no constraints could be obtained since no X-ray monitor was observing during the first minutes-hours. Nevertheless, almost all the proposed models predict fluxes that can be detected by the SXI up to $\sim 0.2 \mathrm{Gpc}$ (Stratta et al. 2018). The XGIS will detect and localize 30-40 onaxis sGRBs yr ${ }^{-1}$ up to $z \sim 1$, while off-axis sGRB as the 170817 event will be detected up to $\sim 70 \mathrm{Mpc}$ (Stratta et al. 2018). Thus, the IRT follow-up of sGRB-KN events 170817 -like will be mainly driven by the SXI detection rate. In summary, THESEUS will detect in principle 2-40 sGRB-KN events 170817-like per years both in X-rays with SXI and optical/NIR with IRT.

\section{CONCLUSIONS}

The discovery of the $\mathrm{KN}$ associated with GRB 170817A/ GW170817 has provided the community with a number of 'foods for thought', and some of them have been examined in this paper.

(i) We find an 'observed' rate of sGRBs associated with $\mathrm{KNe}$ of $352_{-281}^{+810} \mathrm{Gpc}^{-3} \mathrm{yr}^{-1}$, which is definitely larger, by 1-2 orders of magnitude, than most of the estimates reported in Table 2 (all rates have been normalized to $f_{\mathrm{b}}^{-1}=1$ ). This rate is in relatively good agreement with the value of $190_{-160}^{+440} \mathrm{Gpc}^{-3} \mathrm{yr}^{-1}$ found by Zhang et al. (2018). The difference between the two estimates is mainly due to different Dmax adopted in the papers: $49 \mathrm{Mpc}$ versus $65 \mathrm{Mpc}$ (Zhang et al. 2018). From a theoretical point of view, these high rates can be reproduced for an appropriate choice of the minimum luminosity of the luminosity function, i.e. $\sim 10^{47}$ erg rather than $10^{50-51} \mathrm{erg}$ (see equation 1 and Fig. 1). As an alternative one should consider the possibility that GRB 170817A might be the prototype of a class of peculiar/sub-luminous sGRBs, associated with KNe, which not necessarily form the main bulk of the sGRBs population. For example, the sub-luminous SN 1987A that was discovered in the 'local' Universe belongs to a relatively tiny class, $\lesssim 10$ per cent (Pastorello et al. 2004) of intrinsically rare sub-luminous Type II $\mathrm{SNe}$.

(ii) The comparison between the value of 'observed' $\mathrm{KN}$ rate presented in this paper with the much higher density of BNS mergers estimated by the LVC (Abbott et al. 2017a) points out that either a minor fraction of BNS mergers produce sGRBs characterized by an isotropic emission or most BNS mergers produce beamed sGRBs that can be observed under viewing angles as large as $\theta \sim 40^{\circ}$. This second scenario is supported by Mooley et al. (2018), who find for GRB 170817A a $\theta \sim 5^{\circ}$ collimated jet that was likely observed from a viewing angle of $\sim 20_{-5}^{+5}$.

(iii) An 'observed' rate of $\sim 352$ events per $\mathrm{Gpc}^{-3} \mathrm{yr}^{-1}$ offers excellent perspectives for the identification of the electromagnetic counterpart of BNS events detected during next LIGO/Virgo observing runs. On the basis of our analysis, we estimate that future optical surveys will be able to detect, within $200 \mathrm{Mpc}, N_{\mathrm{KN}}=f_{\mathrm{b}}^{-1} \times 12_{-10}^{+27}$

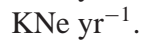

(iv) The relatively high frequency of occurrence of $\mathrm{KNe}$ will allow to some of the future facilities the direct detection of $\mathrm{KNe}$ without GW triggers. Particularly, LSST will have the capability to discover dozens to hundreds of KNe up to $\mathrm{z} \sim 0.25$, that is well beyond the current advanced LIGO/Virgo capabilities. An opposite trend is shown by SKA. Preliminary estimates indicate that this array of radio telescopes will have the capability to carry out wonderful follow-ups for KNe discovered by other facilities, while it will not be so efficient in discovering $\mathrm{KNe}$ during 'blind' and 'shallow' radio surveys.

\section{ACKNOWLEDGEMENTS}

MDV would like to thank the Instituto de Astrofisica de Andalucía for its hospitality and creative atmosphere. The authors thank the referee for her/his comments that have helped to improve the presentation of the data. We are also indebted to Pia Astone for her critical reading of the manuscript.

\section{REFERENCES}

Abbott B. P. et al., 2017a, Phys. Rev. Lett., 119, 161101

Abbott B. P. et al., 2017b, ApJ, 848, L12

Abbott B. P. et al., 2018, Living Rev. Relativ., 21, 3 
Amati L. et al., 2018, Adv. Sp. Res., 62, 191

Ando S., 2004, ApJ, 607, 20

Arcavi I. et al., 2017, Nature, 551, 64

Belczynski K., Kalogera V., Bulik T., 2002, ApJ, 572, 407

Belczynski K., Kalogera V., Rasio F. A., Taam R. E., Zezas A., Bulik T., Maccarone T. J., Ivanova N., 2008, ApJS, 174, 223

Belczynski K., Repetto S., Holz D. E., O’Shaughnessy R., Bulik T., Berti E., Fryer C., Dominik M., 2016, ApJ, 819, 108

Belczynski K., Taam R. E., Kalogera V., Rasio F. A., Bulik T., 2007, ApJ, 662,504

Bellm E., Kulkarni S., 2017, Nat. Astron., 1, 71

Benacquista M., Downing J., 2013, Living Rev. Relativ., 16, 4

Beniamini P., Hotokezaka K., Piran T., 2016, ApJ, 832:149

Berger E., 2014, ARA\&A, 52, 43

Bogomazov . A. I., Lipunov V. M., Tutukov A. V., 2007, Astron. Rep., $51: 308$

Brocato E. et al., 2018, MNRAS, 474, 411

Bromberg O., Nakar E., Piran T., Sari R., 2013, ApJ, 764, 179

Burgay M. et al., 2003, Nature, 426, 531

Cantiello M. et al., 2018, ApJ, 856, 126

Cappellaro E. et al., 2015, A\&A, 584, A62

Chruslinska M., Belczynski K., Klencki J., Benacquista M., 2018, MNRAS, 474, 2937

Coulter D. A. et al., 2017, Science, 358, 1556

Coward D. M. et al., 2012, MNRAS, 425, 2668

Cowperthwaite P. S. et al., 2017, ApJ, 848, L17Ghir

Doctor Z. et al., 2017, ApJ, 837, 57

Dominik M. et al., 2015, ApJ, 806, 263

D’Avanzo P., 2015, J. High Energy Astrophys., 7, 73

Eichler D., Livio M., Piran T., Schramm D. N., 1989, Nature, 340, 126

Fong W., Berger E., Margutti R., Zauderer B. A., 2015, ApJ, 815, 102

Gehrels N., 1986, ApJ, 303, 336

Gehrels N. et al., 2005, Nature, 437, 851

Ghirlanda G. et al., 2016, A\&A, 594, A84

Grado A. et al., 2018, to be published in the Proceedings of the THESEUS Workshop 2017 Journal of the Italian Astronomical Society (Mem.SAIt). preprint (arXiv: 1802.01692)

Granot J., Guetta D., Gill R., 2017, ApJ, 850, L24

Guetta D., Piran T., 2005, A\&A, 435, 421

Guetta D., Piran T., 2006, A\&A, 453, 823

Guetta D., Stella L., 2009, A\&A, 498, 329

Hulse R. A., Taylor J. H., 1975, ApJ, 195, L51

Jin Z. P., Li X., Cano Z., Covino S., Fan Y. -Z., Wei D. -M., 2015, ApJ, 811, L22

Jin Z. P. et al., 2018, ApJ, 857, 128

Kalogera V., Narayan R., Spergel D. N., Taylor J. H., 2001, ApJ, 556, 340

Kalogera V. et al., 2004, ApJ, 601, L179

Kruckow M. U., Tauris T. M., Langer N., Kramer M., Izzard R. G., 2018, MNRAS, 481, 1908

Lattimer J. M., Schramm D. N., 1976, ApJ, 210, 549
Lazzati D., López-Cámara D., Cantiello M., Morsony B. J., Perna R., Workman J. C., 2017, ApJ, 848, L6

Levan A. J. et al., 2017, ApJ, 848, L28

Li L. -X., Paczyński B., 1998, ApJ, 507, L59

Lipunov V. M. et al., 2017, ApJ, 850, L1

Mapelli M., Giacobbo N., 2018, MNRAS, 479, 4391

Margutti R. et al., 2018, ApJ, 856, L18

Matteucci F., Romano D., Arcones A., Korobkin O., Rosswog S., 2014, MNRAS, 438, 2177

Meegan, C. et al. 2009, ApJ, 702:791

Metzger B. et al., 2010, MNRAS, 406, 2650

Mooley K. P. et al., 2018, Nature, 554, 207

Nakar E., Gal-Yam A., Piran T., Fox D. B., 2006, ApJ, 640, 849

Nakar E., Piran T., 2011, Nature, 478, 82

Nakar E., Piran T., 2018, MNRAS, 478, 407

Narayan R., Piran T., Kumar P., 2001, ApJ, 557, 949

Narayan R., Piran T., Shemi A., 1991, ApJ, 379, L17

Pastorello A. et al., 2004, MNRAS, 347, 74

Perna R., Belczynski K., 2002, ApJ, 570, 252

Petrillo C. E., Dietz A., Cavaglià M., 2013, ApJ, 767, 140

Phinney E. S., 1991, ApJ, 380, L17

Pian E. et al., 2017, Nature, 551, 67

Piran T., 1992, ApJ, 389, L45

Pérez-Torres M., et al., 2015, Proceedings of Advancing Astrophysics with the Square Kilometre Array (AASKA14), SISSA MediaLab, Trieste, p. 60

Rezzolla M., Giacomazzo B., Baiotti L., Granot J., Kouveliotou C., Aloy M. A., 2011, ApJ, 732, L6

Sadowski A., Belczynski K., Bulik T., Ivanova N., Rasio F. A., O’Shaughnessy R., 2008, ApJ, 676, 1162

Savchenko V. et al., 2017, ApJ, 848, L15

Smartt S. J. et al., 2017, Nature, 551, 75

Stratta G. et al., 2018, Adv. Space Res., 62, 662

Tanaka M., Hotokezaka K., 2013, ApJ, 775, 113

Tanvir N. R., Levan A. J., Fruchter A. S., Hjorth J., Hounsell R. A., Wiersema K., Tunnicliffe R. L., 2013, Nature, 500, 547

Tanvir N. R. et al., 2017, ApJ, 848, L27

Tonry J. L., 2011, PASP, 123, 58

Troja E. et al., 2017, Nature, 551, 71

Valenti S. et al., 2017, ApJ, 848, L24

Vangioni E., Goriely S., Daigne F., François P., Belczynski K., 2016, MNRAS, 455, 17

Wanderman D., Piran T., 2015, MNRAS, 448, 3026

Yang S. et al., 2017, ApJ, 851, L48

Zhang B. B. et al., 2018, Nat. Co., 9, 447

Ziosi B. M., Mapelli M., Branchesi M., Tormen G., 2014, MNRAS, 441, 3703

This paper has been typeset from a $\mathrm{T}_{\mathrm{E}} \mathrm{X} / \mathrm{LT} \mathrm{E} \mathrm{X}$ file prepared by the author. 